\title{
MOTIVACIONES PARA LA ACTIVIDAD FÍSICA DEPORTIVA Y CONSUMO DE ALCOHOL EN ESTUDIANTES DE PREPARATORIA
}

\author{
MOTIVATIONS FOR SPORTS ACTIVITIES AND \\ ALCOHOL USE IN HIGH SCHOOL STUDENTS
}

\author{
Arodi Tizoc Márquez \\ María Magdalena Alonso-Castillo ${ }^{* *}$ \\ Marco Vinicio Gómez Meza ${ }^{* * *}$ \\ Bertha Alicia Alonso Castillo ${ }^{* * * *}$ \\ Nora Nelly Oliva Rodríguez ${ }^{* * * *}$ \\ Nora Angélica ArmendÁRIZ-GarCíA ${ }^{* * * * *}$
}

\begin{abstract}
RESUMEN
La actividad física deportiva se ha considerado un factor protector de consumo de alcohol, no obstante la relación de estas variables no es consistente. Objetivo: Identificar la relación entre las motivaciones para la actividad física deportiva, días y minutos utilizados y el consumo de alcohol en adolescentes. Material y método: Diseño descriptivo correlacional con muestreo aleatorio estratificado, con asignación proporcional al tamaño del estrato. En cada estrato se usó muestreo por conglomerados unietápico. La muestra fue de 415 adolescentes, con poder de 0,90. Se aplicó Escala de Medida de los Motivos para la Actividad Física-Revisada [MPAM-R], Cuestionario de Identificación de los Trastornos debidos al Consumo de Alcohol [AUDIT]. Resultados. Los días $(\mathrm{rs}=0,254, \mathrm{p}<0,001)$ y minutos $(\mathrm{r} s=0,253, \mathrm{p}<0,001)$ dedicados a la actividad física deportiva relacionaron positivamente con las motivaciones. Se identificó una relación positiva entre motivaciones intrínsecas para la actividad física deportiva y consumo sensato de alcohol ( $\mathrm{p}=0,049)$. Así también, los días $(\mathrm{r}=0,253, \mathrm{p}<0,001)$ y minutos $(\mathrm{rs}=0,270, \mathrm{p}<0,001)$ utilizados en la actividad física deportiva relacionaron positivamente con el consumo de alcohol. Conclusión: Las motivaciones se asociaron con los días y minutos dedicados a la actividad física deportiva y con el consumo sensato de alcohol. Los días y minutos dedicados a la actividad física deportiva también presentaron una relación positiva con el consumo de alcohol. Se propone desarrollar modelos predictivos, que sean la base de futuras intervenciones de enfermería enfocadas a la promoción de la salud y prevención del consumo de alcohol, mediante estrategias de motivación de la actividad física deportiva.
\end{abstract}

Palabras clave: Motivación, estudiantes, deportes, consumo de bebidas alcohólicas, enfermería psiquiátrica.

\footnotetext{
ABSTRACT

Alcohol use and abuse is a public health problem, it is a causal factor for more than 200 diseases and injuries; sports activity has been considered a protective factor for alcohol use, however, the relationship of these variables

* Maestría en Ciencias de Enfermería, Profesor, Universidad Autónoma de Baja California. México. E-mail: atizoc@uabc.edu.mx

** Doctora en Filosofía. Profesora de la Facultad de Enfermería, Universidad Autónoma de Nuevo León, México. Email: magdalena_alonso@hotmail.com.

*** Ph.D. Profesor Universidad Autónoma de Nuevo León, México. mgomez@ccr.dsi.uanl.mx

${ }^{* * * *}$ Doctora en Bioética. Profesora de la Facultad de Enfermería, Universidad Autónoma de Nuevo León, México. Correo electrónico: balonso_mx@yahoo.com.mx

Maestría en Ciencias de Enfermería, Profesora, Facultad de Enfermería, Universidad Autónoma de Nuevo León, México. E-mail:norisnelly@gmail.com

${ }_{* * * * * *}$ Doctora en Ciencias de Enfermería, Profesora de la Facultad de Enfermería, Universidad Autónoma de Nuevo León, México. Email: nordariz@hotmail.com
} 
is not consistent. Objective: To identify the relationship between motivations for sports activity, measuring days and minutes dedicated to it, and alcohol use in teenagers. Methods: A descriptive correlational design with stratified random sampling and proportional allocation to the stratum size was used. A single stage cluster sampling was conducted for each stratum. The sample included 415 teenagers with a power of 0.90 . The Scale of Motives for Physical Activity Measurement-Revised [MPAM-R] and the Alcohol Use Disorders Identification Test [AUDIT] were used. Results: The number of days $(\mathrm{rs}=0.254, \mathrm{p}<0.001)$ and minutes $(\mathrm{rs}=0.253, \mathrm{p}<0.001)$ spent in sports activities by teenagers were positively and significantly correlated with alcohol use [AUDIT]. Likewise, the number of days $(\mathrm{r}=0.253, \mathrm{p}<0.001)$ and minutes $(\mathrm{rs}=0.270, \mathrm{p}<0.001)$ spent in sports activities were positively and significantly correlated with motivations. Conclusions: The number of days and minutes spent in sports activities by teenagers is related to alcohol use and motivations. There is a need for predictive models as a basis for future nursing interventions focused on health promotion and alcohol use prevention by using strategies that promote sports activities.

Key words: Motivation, students, sports, alcohol drinking, psychiatric nursing.

Fecha recepción: 09/03/15 Fecha aceptación: 18/08/16

\section{INTRODUCCIÓN}

El uso y abuso de alcohol, de acuerdo con la Organización Mundial de la Salud (OMS), es parte de la agenda de salud de los gobiernos; en virtud de que es un factor causal de más de 200 enfermedades y lesiones (1). Además, se asocia al riesgo de padecer enfermedades mentales y del comportamiento, incluyendo la dependencia al alcohol, enfermedades no transmisibles como las cardiovasculares, cirrosis hepática, algunos tipos de neoplasias, $\mathrm{y}$ traumatismos derivados de violencia y accidentes de tránsito. Se considera además un problema de salud pública de gran trascendencia social ya que se calcula que ocurren cada año 320 mil defunciones de adolescentes con edades de 15 y 29 años en el mundo por causa del consumo de alcohol (2).

En México, en el año 2011 el 42,9\% de los adolescentes de 12 a 17 años de edad habían consumido alcohol alguna vez en su vida, el $46 \%$ corresponde a hombres y el $39,7 \%$ a mujeres, en tanto la prevalencia en el último año de consumo de alcohol fue de $30 \%$ (31,8\% hombres y $28,1 \%$ mujeres). En los años de 2002 a 2011 el índice de consumo diario de alcohol en adolescentes se mantu- vo estable con 1,4\%, el índice de consumo de alcohol dependiente en adolescentes registró un incremento significativo, que aumentó de 2,1 a $4,1 \%$, mientras que el consumo alto del 2008 al 2011 se mantuvo con la misma proporción que fue de $32 \%$ (3).

Por otra parte, el consumo de alcohol como práctica social tiene una carga cultural que se transmite por generaciones y está presente en diversos ámbitos de la vida del ser humano. En la época actual esta práctica se encuentra presente en todos los sectores y grupos de la sociedad como en los adolescentes $(4,5)$.

La adolescencia es una etapa de transición donde se experimentan una serie de cambios en el crecimiento físico, desarrollo psicológico y social; además en esta etapa los adolescentes tanto hombres como mujeres amplían el número de grupos de referencia social con los que interactúan y socializan como amigos, compañeros de aula, grupos sociales, religiosos y deportivos, los que pueden tener comportamientos de riesgo que pueden ser reproducidos socialmente, como el consumo de alcohol, sin importar el género al que pertenezcan (6). Se ha observado que el consumo de alcohol a temprana edad afectará la vida futura de los adolescentes. En el corto 
plazo pueden desarrollar mayor tolerancia al alcohol, así como un gran sufrimiento en la familia y lo anterior se reflejará en el incremento de los costos del sistema de salud en razón de hospitalizaciones, mayores consultas relacionadas de forma directa o indirecta con el consumo $(7,8)$.

Es importante resaltar que la OMS en su Estrategia mundial sobre régimen alimentario, actividad física y salud indica que la actividad física puede contribuir en el desarrollo psicosocial de los adolescentes y jóvenes, dado que brinda la oportunidad para expresarse y fomenta la autoconfianza, la interacción social, mejora el rendimiento escolar y la integración. También se ha sugerido que los adolescentes y jóvenes activos pueden adoptar con más facilidad otros comportamientos saludables, como evitar el consumo de alcohol, tabaco y drogas ilícitas. Por lo anterior se convierte la actividad física deportiva en un factor de protección de consumo de alcohol y otras drogas, porque contribuye al desarrollo de valores personales y sociales, introducen normas de comportamiento, pone a prueba los límites personales y grupales, y existe una mejora de las capacidades personales y de competición (9). Sin embargo, esta relación no está totalmente clara, dado que algunos estudios muestran que la actividad física deportiva evita o reduce el consumo de alcohol, no obstante en otros estudios no se ha encontrado dicha relación significativa $(10,11)$.

Navarro et al. (12) señalan que las conductas se inician, se mantienen o se abandonan como consecuencia de la motivación del adolescente. Esto se puede explicar desde la teoría de la autodeterminación, la cual considera que la motivación puede surgir de forma interna o externa a la persona, estableciendo diferentes formas de motivación (intrínseca y extrínseca) que funcionan como activadores del desarrollo cognitivo y social. Se centra en cómo los factores culturales y sociales son centrales al facilitar o dificultar el sentido de voluntad e iniciativa personal en la realización de una conducta (13), referida en el presente estudio al consumo de alcohol y la realización de actividad física deportiva. Las motivaciones intrínsecas se relacionan con la necesidad de explorar el entorno, la curiosidad y obtener placer; las extrínsecas intentan satisfacer demandas externas, ya sea para obtener un premio, recompensa, y por influencia de personas con quienes interactúan (13).

La actividad física deportiva se define por Ries (14) como toda aquella acción corporal del organismo mediante el movimiento que genera gasto energético que ocurre mediante la participación libre y organizada, que tiene como objetivo el bienestar, así como la diversión en el tiempo libre, la creación de nuevas relaciones, y la obtención de resultados competitivos. Esta es de dos tipos: la colectiva y la individual. La colectiva implica una acción corporal a través del movimiento, y cooperación entre dos o más compañeros, la participación puede ser de alternancia o simultánea como lo son el básquetbol, béisbol, boliche, fútbol, softbol entre otros. La individual es la acción corporal mediante el movimiento que realiza una persona, y se puede realizar en un espacio fijo o fluctuante tales como el atletismo, ciclismo, frontón, natación y tenis. Así mismo se ha propuesto que la actividad física deportiva es un medio de abstinencia para el consumo de alcohol en adolescentes $(14,15)$.

Algunos autores han reportado que las conductas como el consumo de alcohol y la realización de la actividad física deportiva pueden ser influidas por las motivaciones intrínsecas y extrínsecas para la actividad física deportiva que se desprenden de la teoría de autodeterminación $(9,13,16)$, las cuales han mostrado relación significativa entre los adolescentes que consumen y no consumen alcohol, siendo más altas las motivaciones intrínsecas y extrínsecas para la actividad física deportiva en quienes no consumen alcohol que en los que sí consumen $(16,17)$, sin embargo los hallazgos no son consistentes entre la presencia o no de una relación significativa de la actividad física deportiva y el menor consumo de alcohol en adolescentes y jóvenes $(10,11,18)$. 
Por lo anteriormente planteado esta temática resulta de interés debido a que la mayoría de los programas de promoción de la salud y de prevención del consumo de alcohol dirigidos a los adolescentes incluyen el desarrollo de la actividad física deportiva para mantener la salud y el bienestar. Sin embargo, se ha observado que los adolescentes y jóvenes que realizan la actividad física deportiva no se encuentran libres del consumo de alcohol.

El estudio aporta al conocimiento científico sobre la relación de las motivaciones para la actividad física deportiva con el tipo de actividad física deportiva (individual o colectiva) y con el consumo de alcohol. Así mismo este conocimiento podrá ser base de futuras intervenciones profesionales de enfermería para la promoción de la salud, enfocada en la actividad física deportiva y para la prevención del consumo de alcohol en adolescentes.

Por tal motivo los objetivos del presente estudio son: 1) determinar la prevalencia y tipo de consumo de alcohol por edad y sexo, 2) identificar las motivaciones intrínsecas y extrínsecas para la actividad física deportiva por tipo de actividad física deportiva, 3) identificar la relación entre las motivaciones para la actividad física deportiva, días y minutos utilizados y el consumo de alcohol en adolescentes.

\section{MATERIAL Y MÉTODO}

El diseño del presente estudio fue descriptivo correlacional, la población se conformó por 4.719 adolescentes de dos instituciones de nivel medio superior del municipio de San Nicolás de los Garza, Nuevo León. Para calcular el tamaño de la muestra se utilizó el programa n`Query Advisor Versión 4.0 ${ }^{\circledR}$, considerando que se deseaba contrastar una hipótesis nula de correlación con un nivel de significancia de 0.05 y potencia de 0.90 cuando el verdadero valor del parámetro era 0.18 , resultando que se requerían de 321 ado- lescentes. Considerando una tasa de no respuesta de $10 \%$, y un efecto de diseño de 1.15 , se requirió un total de 415 adolescentes.

El muestreo fue aleatorio estratificado con asignación proporcional al tamaño del estrato, dentro de cada estrato se usó el muestreo por conglomerados unietápico; el conglomerado se constituyó por cada uno de los grupos escolares de las dos instituciones, obteniéndose un total de 10 grupos seleccionados aleatoriamente.

Para este estudio se utilizó una cédula de datos personales de actividad física deportiva y prevalencia de consumo de alcohol para recabar información sobre datos personales como sexo, edad, la actividad física deportiva que practica, las prevalencias del consumo de alcohol global, lápsica, actual e instantánea y consumo excesivo ocasional (binge drinking).

Para conocer las motivaciones intrínsecas y extrínsecas para la actividad física deportiva se utilizó la Escala de Medida de los Motivos para la Actividad Física-Revisada (19), este instrumento es una versión modificada al idioma español de la escala Motives for Physical Activities Measure [MPAM-R] (20). Esta se compone por 30 reactivos, agrupados en cinco subescalas, estas a su vez se ubican en los dos tipos de motivación intrínseca y extrínseca. En la dimensión de motivación intrínseca para la actividad física deportiva se incluyen las subescalas de disfrute y competencia; las otras tres subescalas: social, salud y apariencia reflejan la dimensión de la motivación extrínseca para la actividad física deportiva.

De acuerdo a lo estipulado por Hodge y Gillespie (21) la escala Likert puede reducir sus opciones de respuesta de 7 a 5 , o de 5 ó 3 alternativas con la finalidad de que el participante no muestre dificultades para responder, cuando son menores de edad o cuando la escolaridad sea menor a 14 años. Por esta razón en este estudio, dadas las características de la muestra, la escala de Likert se redujo de siete a cinco puntos; donde: $1=$ Totalmente en desacuerdo, 2 = En des- 
acuerdo, $3=\mathrm{Ni}$ en desacuerdo, ni de acuerdo, $4=$ De acuerdo y $5=$ Totalmente de acuerdo, donde el valor mínimo es 30 y el valor máximo es 150, lo cual significa que a mayor puntaje mayor motivación intrínseca y extrínseca en el adolescente. Para determinar si está motivado intrínsecamente el valor mínimo es 13 y máximo es 65 . Así mismo para determinar si está motivado extrínsecamente el valor mínimo es 17 y el valor máximo es 85 .

La confiabilidad de la escala total [MPAM-R] reportó un Alpha de Cronbach de 0,82 en población adolescente española y en población mexicana, también presentó consistencia interna aceptable $(\alpha=0,81)$, presentando para la dimensión de motivación intrínseca un coeficiente de 0,87 y para la de motivación extrínseca 0,84 . Así mismo este instrumento fue validado en población mexicana (22), utilizando análisis factorial confirmatorio, mostrando adecuados índices de ajuste.

Para conocer el tipo de consumo de alcohol de los adolescentes se utilizó el Cuestionario de Identificación de los Trastornos debidos al Consumo de Alcohol [AUDIT] (23) validado para la población mexicana (24). El cuestionario consta de 10 reactivos que examinan el consumo de alcohol y sus consecuencias durante los últimos doce meses. La especificidad del AUDIT se sitúa en valores superiores de 0,80 (25). Un estudio que ha utilizado este instrumento en adolescentes de preparatoria de Nuevo León reportó un Alpha de Cronbach de 0,84 (25), para este estudio el cuestionario AUDIT reportó un Alpha de Cronbach de 0,75.

Se obtuvo la autorización de los Comités de Ética e Investigación de la institución donde son procedentes los autores, así como de las instituciones educativas donde se realizó la recolección de datos del presente estudio.

A los grupos de estudiantes seleccionados, mediante las listas oficiales de la institución educativa, se les localizó en cada aula de clase y se les citó en un horario determinado en el aula que se había asignado previamente por las autoridades de la institución para informar sobre el estudio. Una vez reunidos se procedió a explicar de forma clara y sencilla los objetivos de la investigación, los instrumentos de recolección y el tiempo que se requiere para contestarlos. Se invitó a participar en el estudio a todos los estudiantes de los grupos seleccionados, especificando que aquellos estudiantes que no desearan participar tendrían toda la libertad de negarse o retirarse, en cualquier momento del estudio, sin que esto afecte su condición dentro de la institución. Así mismo se precisó que toda la información que se obtuviera de su participación es confidencial y anónima.

Se autorizó la participación en el estudio por parte de los padres de familia o tutores mediante consentimiento informado y el asentimiento informado del estudiante por ser menores de edad. La presente investigación se apegó a lo dispuesto en el Reglamento de la Ley General de Salud en Materia de Investigación para la Salud (26).

Los datos obtenidos fueron procesados con el programa estadístico Statistical Package for the Social Sciences (SPSS) versión 20.0 para Windows. Se empleó estadística descriptiva a través de frecuencias y proporciones, así como estadística inferencial. Se realizó la prueba de normalidad de Kolmogorov-Smirnov con Corrección de Lilliefors para determinar la normalidad de la distribución de las variables continuas y numéricas; en base a los resultados se decidió el uso de estadística no paramétrica (U de Mann-Whitney, $\mathrm{H}$ de Kruskal-Wallis y Coeficiente de Correlación de Spearman) debido a que los datos no presentaron normalidad.

\section{RESULTADOS}

\section{Características sociodemográficas}

Un poco más de la mitad de los participantes $(51,1 \%)$ fueron del sexo femenino, la edad 
de 15 años fue la que predominó entre los participantes. Por otro lado, se observó que el 69,9\% de los adolescentes realizan alguna actividad física deportiva, y el 32,3\% la practican de 2 a 3 días y en menor proporción $(23,6 \%)$ la practican de 4 a 5 días. Respecto al tiempo en minutos que le dedican en un día a la actividad física deportiva, el 15,4\% de los adolescentes mencionó realizar la actividad física deportiva en un período de 15 a 30 min, el $25,1 \%$ refirieron que practican de 30 a 60 min y $18,6 \%$ refirieron que practican de 31 a $90 \mathrm{~min}$.

Se identificó que el tipo de actividad física deportiva individual es la más practicada con un 40,5\%; dentro de esta actividad física deportiva individual, lo que más se practica es caminar/correr con un $17,3 \%$, mientras que la actividad física deportiva colectiva solo el $29,4 \%$ de los participantes la realizan, siendo el fútbol soccer con un 12,0\% el más practicado por los participantes.

\section{Consumo de alcohol}

En cuanto a la prevalencia de consumo de alcohol de los participantes, el 70\% (IC 95\% $[66,75])$ han consumido alcohol alguna vez en la vida, el 47\% (IC 95\% [42, 52]) en el último año, el 27\% (IC 95\% [22,31]) en el último mes, además de que el 12\% (IC 95\% [8,
15]) en los últimos siete días. Así mismo se observó que el consumo de alcohol excesivo ocasional, que se caracteriza por el consumo en varones de cinco a más bebidas alcohólicas y de cuatro a más bebidas en mujeres en un periodo de dos horas (binge drinking), se encuentra presente en el 10\% (IC 95\% [7, 13]) de los participantes que consumen alcohol.

Las prevalencias de consumo de alcohol en el último mes, en los últimos siete días y el consumo excesivo ocasional (binge drinking) fueron más altas en los adolescentes del sexo masculino (34, 16 y 15\%, respectivamente) que en el femenino (19, 8 y $6 \%$, respectivamente) de forma significativa $(p<0,05)$. No obstante, en las prevalencias de consumo de alcohol alguna vez en la vida y en el último año no se observaron diferencias significativas $(p>0,05)$ debido a que las proporciones fueron muy similares en los chicos y las chicas.

En la Tabla 1 se muestra que los adolescentes de 16 a 17 años reportaron significativamente más altas proporciones en las prevalencias de consumo de alcohol alguna vez en la vida (77\%), último mes (34\%), últimos siete días $(18 \%)$ y en el consumo excesivo (binge drinking, 15\%) en comparación con los chicos de 14 a 15 años de edad $(66,21,7$ y $7 \%$, respectivamente).

Tabla 1. Prevalencia de consumo de alcohol en adolescentes participantes del estudio.

\begin{tabular}{|c|c|c|c|c|c|c|c|c|c|c|}
\hline \multirow[b]{2}{*}{ Edad } & \multicolumn{2}{|c|}{$\begin{array}{l}\text { Alguna vez en } \\
\text { la vida }\end{array}$} & \multicolumn{2}{|c|}{$\begin{array}{l}\text { En el último } \\
\text { año }\end{array}$} & \multicolumn{2}{|c|}{$\begin{array}{c}\text { En el } \\
\text { último mes }\end{array}$} & \multicolumn{2}{|c|}{$\begin{array}{l}\text { En los últimos } \\
7 \text { días }\end{array}$} & \multicolumn{2}{|c|}{$\begin{array}{c}\text { Consumo } \\
\text { excesivo } \\
\text { ocasional }\end{array}$} \\
\hline & $f$ & $\%$ & $f$ & $\%$ & $f$ & $\%$ & $f$ & $\%$ & $f$ & $\%$ \\
\hline 14 a 15 años & 160 & 66 & 107 & 44 & 52 & 21 & 17 & 7 & 18 & 7 \\
\hline 16 a 17 años & 132 & 77 & 89 & 52 & 58 & 34 & 31 & 18 & 25 & 15 \\
\hline$\chi^{2}$ & \multicolumn{2}{|c|}{5,73} & \multicolumn{2}{|c|}{2,4} & \multicolumn{2}{|c|}{7,84} & \multicolumn{2}{|c|}{11,9} & \multicolumn{2}{|c|}{5,5} \\
\hline$p$ & \multicolumn{2}{|c|}{0,011} & \multicolumn{2}{|c|}{0,073} & \multicolumn{2}{|c|}{0,004} & \multicolumn{2}{|c|}{0,001} & \multicolumn{2}{|c|}{0,015} \\
\hline
\end{tabular}

Nota: $\mathrm{n}=$ muestra total, $\chi 2=$ Estadístico Chi-Cuadrada de Pearson, $\mathrm{p}=$ significancia. 
El tipo de consumo de alcohol sensato predomina en adolescentes del sexo femenino $(78,9 \%)$, mientras que para el consumo de alcohol dependiente $(21,8 \%)$ y dañino $(25,7 \%)$ es mayor en el sexo masculino en comparación con el femenino (12,7 y 8,4\%, respectivamente), mostrándose diferencias significativas $(p=0,001)$.

\section{Motivaciones para la actividad física depor- tiva por tipo de actividad física deportiva}

Respecto a las motivaciones intrínsecas para la actividad física deportiva por el tipo de actividad física realizada por los adolescentes, se encontró diferencia significativa de los motivos de disfrute $(U=7349.5, p<0,001)$, siendo las medianas más altas en los adolescentes que practicaban la actividad física deportiva colectiva; los motivos de competencia no reportaron diferencias significativas. Por otro, lado en las motivaciones extrínse- cas, los motivos sociales tuvieron medianas significativamente más altas $(\mathrm{Mdn}=70,0)$ en los adolescentes que practicaban la actividad física deportiva colectiva, los motivos de salud y de apariencia presentaron medianas significativamente más altas $(\mathrm{p}<0,01)$ en los adolescentes que practicaban actividad física deportiva de manera individual (Tabla 2).

En lo referente a la actividad física deportiva (días y minutos dedicados) se identificaron correlaciones positivas y significativas $(\mathrm{p}<0,001)$ con las motivaciones intrínsecas y extrínsecas para la actividad física deportiva (Tabla 3).

Se identificó la relación entre motivaciones y consumo de alcohol; en el caso de las motivaciones intrínsecas para la actividad física deportiva, ésta se correlacionó positivamente con el consumo sensato de alcohol $\left(r_{s}\right.$ $=0,163, p=0,049)$. Esto significa que a mayor motivación intrínseca mayor es el consumo de alcohol sensato en los adolescentes.

Tabla 2. Motivaciones para la actividad física deportiva por tipo de actividad física deportiva en adolescentes participantes del estudio $(\mathrm{na}=290)$.

\begin{tabular}{lllllll}
\hline $\begin{array}{l}\text { Motivaciones para la actividad } \\
\text { física deportiva }\end{array}$ & $\mathrm{n}$ & Media & Mdn & Estadígrafo & $\mathrm{p}$ \\
\hline
\end{tabular}

\section{Intrínsecas}

Disfrute

$\begin{array}{cccccc}\text { Individual } & 168 & 74,5 & 75 & 7349,5^{\dagger} & 0,001 \\ \text { Colectiva } & 122 & 81,6 & 82,1 & & \end{array}$

Competencia

\begin{tabular}{cccccc} 
Individual & 168 & 68,6 & 70,08 & $9967^{\dagger}$ & 0,888 \\
Colectiva & 122 & 68,9 & 70,8 & 98 \\
\hline
\end{tabular}

\section{Extrínsecas}

Social

$\begin{array}{cccccc}\text { Individual } & 168 & 55,4 & 55 & 6119^{\ddagger} & 0,001 \\ \text { Colectiva } & 122 & 69,1 & 70 & & \end{array}$

Salud

$\begin{array}{cccccc}\text { Individual } & 168 & 81,3 & 83,3 & 8226^{\ddagger} & 0,008 \\ \text { Colectiva } & 122 & 76,7 & 77 & & \end{array}$

Apariencia

$\begin{array}{cccccc}\text { Individual } & 168 & 74,6 & 75 & 7456^{\ddagger} & 0,001 \\ \text { Colectiva } & 122 & 65,7 & 64,5 & \end{array}$

Nota: $\mathrm{na}=$ muestra parcial de los que realizan actividad física deportiva, $\mathrm{Mdn}=$ Mediana, ${ }^{\dagger}=\mathrm{U}$ de Mann-Whitney, ${ }^{\ddagger}=\mathrm{H}$ de Kruskal Wallis, $\mathrm{p}=$ significancia. 
Tabla 3. Relación entre los días y minutos dedicados a la actividad física deportiva con las motivaciones intrínsecas y extrínsecas para la actividad física deportiva en adolescentes participantes del estudio.

\begin{tabular}{|c|c|c|c|c|}
\hline Variable & $\begin{array}{l}\text { Días a la semana } \\
\text { de actividad física }\end{array}$ & $p$ & $\begin{array}{l}\text { Minutos en un día } \\
\text { de actividad física }\end{array}$ & $p$ \\
\hline Motivos para la actividad física & $0,253^{* *} \dagger$ & 0,001 & $0,270^{* *} \dagger$ & 0,001 \\
\hline Motivación intrínseca & $0,190^{* *} \dagger$ & 0,001 & $0,297^{\star *} \dagger$ & 0,001 \\
\hline Disfrute & $0,075 \dagger$ & 0,207 & $0,300^{* *} \dagger$ & 0,001 \\
\hline Competencia & $0,239^{* *} \dagger$ & 0,001 & $0,226^{* *} \dagger$ & 0,001 \\
\hline Motivación extrínseca & $0,246^{\star *} \dagger$ & 0,001 & $0,179^{* *} \dagger$ & 0,002 \\
\hline Social & $0,134^{\star} \dagger$ & 0,023 & $0,259^{* *} \dagger$ & 0,001 \\
\hline Salud & $0,283^{* *} \dagger$ & 0,001 & $0,114 \dagger$ & 0,053 \\
\hline Apariencia & $0,184^{* *} \dagger$ & 0,002 & $0,047 \dagger$ & 0,428 \\
\hline
\end{tabular}

Nota: valor de $\mathrm{p}=$ significancia, ${ }^{\star} \mathrm{p}<0,05 .{ }^{* *} \mathrm{p}<0,01,{ }^{* * *} \mathrm{p}<0,001, \mathrm{nb}=196$ muestra parcial consumo de alcohol en el último año, $\dagger$ Coeficiente de Correlación de Spearman.

También se encontraron correlaciones positivas y significativas $(\mathrm{p}<0,01)$ entre la actividad física deportiva (días y minutos dedicados) con el consumo de alcohol (AUDIT) y el consumo sensato de alcohol; esto indica que los chicos que utilizan más días y minutos para realizar actividad física deportiva presentaron mayor consumo de alcohol (AUDIT) de forma sensata (Tabla 4).

Tabla 4. Relación entre los días y minutos dedicados a la actividad física deportiva y el consumo de alcohol en adolescentes participantes del estudio.

\begin{tabular}{lcccc}
\hline Variable & $\begin{array}{c}\text { Días a la semana de } \\
\text { actividad física }\end{array}$ & $p$ & $\begin{array}{c}\text { Minutos en un día de } \\
\text { actividad física }\end{array}$ & $p$ \\
\hline Puntaje AUDIT (total) & $0,254^{* *} \dagger$ & 0,002 & $0,253^{* *} \dagger$ & 0,002 \\
Índice sensato & $0,315^{* *} \dagger$ & 0,001 & $0,254^{* *} \dagger$ & 0,002 \\
Índice dependiente & $0,149 \dagger$ & 0,075 & $0,182^{*} \dagger$ & 0,03 \\
Índice dañino & $0,114 \dagger$ & 0,177 & $0,163 \dagger$ & 0,052 \\
\hline
\end{tabular}

Nota: valor de $\mathrm{p}=$ significancia, ${ }^{*} \mathrm{p}<0,05 .{ }^{* *} \mathrm{p}<0,01,{ }^{* * *} \mathrm{p}<0,001, \mathrm{nb}=196$ muestra parcial consumo de alcohol en el último año, $\dagger$ Coeficiente de Correlación de Spearman.

\section{DISCUSIÓN Y CONCLUSIÓN}

Diferentes organismos, desde instituciones gubernamentales hasta órganos del sector sa- lud privado o público, afirman que existe alto consumo de alcohol en México por parte de los adolescentes, señalando la problemática que se despliega a causa del consumo de alcohol (27). El presente estudio no documen- 
ta lo contrario, debido a las altas prevalencias de consumo de alcohol reportadas.

No obstante, se observa que los adolescentes de sexo masculino siguen mostrando mayores prevalencias de consumo de alcohol alguna vez en la vida, en el último mes y en los últimos siete días y en el consumo de alcohol excesivo ocasional (binge drinking); estos hallazgos podrían explicarse en las premisas socioculturales que construye la sociedad, donde se acepta como normal la conducta del consumo de alcohol incluso excesivo en el sexo masculino, no así en el femenino. Sin embargo, en los últimos años la mujer está optando por esta conducta debido a la aceptación social entre sus pares, así como a las expectativas positivas que adolescentes y jóvenes comparten acerca de la sustancia, además de considerar este tema como un asunto de igualdad de género $(5,28)$.

Un dato importante de señalar es que la prevalencia del consumo de alcohol aumenta conforme avanza la edad de los adolescentes, esto podría explicarse en función de la temprana edad de inicio al consumo de alcohol, por la fácil adquisición de la sustancia en el entorno social, por la baja percepción de riesgo del consumo, la exposición a la oportunidad y la tentación de probar el alcohol (29).

Respecto al tipo de consumo de alcohol, las adolescentes mexicanas presentan un consumo de alcohol sensato, dicho consumo ha ido aumentando de manera significativa, esto posiblemente ocurre por la presencia de consumo de alcohol en la familia (beber en compañía de los padres, fiestas del hogar y percibir que beber alcohol es una tradición en la familia), dicho acompañamiento parental conlleva a un consumo responsable de alcohol, pero puede conducir hacia el tránsito de un consumo dependiente y dañino $(3,30$, 31). Respecto al tipo de consumo de alcohol dependiente y dañino se observa más alto en adolescentes masculinos, lo anterior puede ser explicado en el sentido de que si bien es cierto que la sociedad gradualmente ha ido aceptando el consumo de alcohol de la mujer, se espera que este consumo sea menor en frecuencia y cantidad que en el hombre. Por otra parte, es frecuente que los adolescentes varones asocien esta conducta de consumo excesivo y dañino como sinónimo de fortaleza, hombría, valentía además que a nivel de los padres de familia esta conducta es aceptada, tolerada y se refuerza con el consumo excesivo de alcohol en adolescentes varones más que en las mujeres. La denominada tolerancia social está influyendo en el incremento de la ingesta del alcohol y en el aumento de los riesgos a la salud de los adolescentes, los cuales muestran una infravaloración o desconocimiento de los peligros del consumo excesivo de alcohol $(5,7,32)$.

Por otra parte, se ratifica en el presente estudio lo reportado por algunos autores acerca de que la motivación intrínseca y extrínseca representan un pilar básico de las conductas saludables y un nexo directo con el tipo de actividad física deportiva individual y colectiva que se práctica, así como con la intensidad de la actividad en función de días y minutos dedicados a esta práctica. De este modo, los resultados mostraron que al incrementarse los motivos para la actividad física deportiva, también aumentó la intensidad de la actividad $(32,33)$.

No obstante, llama la atención en este estudio, que a mayor intensidad de la actividad física deportiva en función de los días y minutos utilizados para realizar esta conducta, mayor es el consumo de alcohol en función de los puntajes del (AUDIT). Esta relación se ha documentado en algunos estudios que muestran que mientras más actividad física deportiva realicen los adolescentes y jóvenes menor es el consumo de alcohol, pero también se ha encontrado que el consumo de alcohol aumenta en adolescentes independientemente de que realicen la actividad física deportiva (34).

Es posible que existan factores comunes entre la actividad física deportiva y el consumo de alcohol, uno de estos puede ser el ocio 
ya que ambos comportamientos se llevan a cabo en los tiempos libres dedicados al descanso, la diversión y la socialización. Algunos autores han afirmado que consumir alcohol significa divertirse, disfrutar, compartir y por ello este ocurre en el contexto de la relación grupal. Por otra parte se ha indicado que quienes realizan actividad física deportiva perciben tener beneficios en su salud. Lo que puede generar confianza en los adolescentes de que la actividad física deportiva puede proteger o contrarrestar los posibles efectos nocivos del alcohol en su salud $(31,32)$.

Específicamente se documentó la existencia de mayores motivaciones intrínsecas (disfrute) cuando los adolescentes realizan actividad física deportiva colectiva, lo anterior concuerda con lo reportado en un estudio en jóvenes colombianos (35) que indican que las motivaciones intrínsecas para la actividad física deportiva (satisfacción-disfrute) incrementa la práctica de la actividad física deportiva, dado que esta explora el entorno, coadyuva a resolver grupalmente la curiosidad, facilita la aceptación social y logro de objetivos colectivos y es placentero, por la convivencia e interacción con sus amigos y pares, la cual refuerza el mantener la actividad física deportiva (19).

Respecto a las motivaciones extrínsecas y el tipo de actividad física deportiva realizada de forma individual y colectiva se documentó que existe diferencia significativa entre los motivos externos de tipo social donde las medias y medianas más altas fueron para la actividad colectiva. En este sentido existe congruencia entre la motivación social y practicar una actividad colectiva dado que se realiza esta actividad para satisfacer demandas externas, para obtener un premio o recompensa, por el deseo de agradar a las personas con quienes interactúan (36). De esta forma parece ser que la realización de actividad física deportiva colectiva se práctica para resolver aspectos de integración y aceptación social que por la competición.

Adicionalmente, las motivaciones extrín- secas como la salud y la apariencia mostraron diferencia significativa con el tipo de actividad física deportiva individual, lo anterior podría explicarse en lo establecido por Deci y Ryan (13), donde las razones que se vinculan con la realización de esta actividad se encuentran en satisfacer una demanda personal de mantenerse sanos, por circunstancias particulares e incluso por obtener reconocimiento. Aunado a lo anterior, en esta etapa de la adolescencia la apariencia significa aceptación de los otros, la salud cobra importancia en función del peso corporal y por ello puede ser que estos adolescentes realicen esta actividad buscando una buena figura o tener una apariencia agradable ante sus pares que les permita socializar y ser aceptados.

En base a lo anterior se puede concluir que el consumo de alcohol es una conducta presente en los adolescentes que estudian preparatoria independientemente del género o la edad de estos, sin embargo los hombres y quienes tienen mayor edad son quienes reportan mayor proporción de consumo de tipo dependiente y dañino. Por otro lado se identificó que los adolescentes que practican actividad física deportiva colectiva reportaron mayores motivaciones intrínsecas de disfrute en la realización de esta actividad. Respecto a las motivaciones extrínsecas, las de tipo social fueron significativamente diferentes, siendo las medias y medianas más altas para actividad física colectiva que para la individual. No obstante, las motivaciones extrínsecas de tipo salud y apariencia también mostraron diferencias, pero estas fueron más altas cuando se practica la actividad física deportiva individual versus la colectiva.

Así mismo se encontró que los motivos para la actividad física deportiva y el consumo de alcohol medido por el AUDIT se relacionaron positiva y significativamente con los días de la semana y los minutos al día que realizan la actividad física deportiva, variables que podrían estarse influenciando respecto a la intensidad de la actividad, por lo que se requiere profundizar en ellas. Por tal 
motivo estos resultados son relevantes para la disciplina de enfermería ya que muestran que se debe incluir en las intervenciones de enfermería de promoción de la salud la actividad física deportiva y los estilos de vida saludables como el no consumo de alcohol en los adolescentes, utilizando estrategias de socialización, esparcimiento, trabajo en grupos, habilidades sociales colectivas que actúen en ambas conductas protegiendo la integridad del adolescente.

Como limitaciones de este estudio se considera que en la selección de la muestra no se incluyó adolescentes escolarizados de área rural, además que la aplicación de los instrumentos fue en un momento en el tiempo (transversal).

\section{REFERENCIAS}

1. World Health Organization (WHO). Global status report on alcohol and health [Internet]. Geneva: WHO; 2014 [citado 20 nov 2014]. 376 p. Disponible en: http://apps.who.int/iris/bitstre am/10665/112736/1/9789240692763_ eng.pdf

2. Anderson P, Gual A, Colon J. Alcohol y atención primaria de la salud: informaciones clínicas básicas para la identificación y el manejo de riesgos y problemas [Internet]. Washington: OPS/OMS; 2008 [citado 20 nov 2014]. 139 p. Disponible en: http://www.who.int/substance_abuse/publications/alcohol_atencion_primaria.pdf

3. Secretaría de Salud, Comisión Nacional contra las Adicciones [CONADIC]. Programa de Acción Específico. Prevención y Tratamiento de las Adicciones. Actualización 2011-2012. México, D.F.: Secretaría de Salud; 2011.48 p.

4. Taylor M, Walker T, Austin C, Thoth C, Welch D. The Influence of Cultural Identification, Religiosity, and Self-Esteem on
Alcohol Use Among African American, Hispanic, and White Adolescents. West J Black Stud. 2011; 35(2): 139-156.

5. Alonso-Castillo MM, Álvarez-Bermúdez J, López-García K, Rodríguez-Aguilar L, Alonso-Castillo MT, Armendáriz N. Factores de riesgo personales, psicosociales y consumo de alcohol en mujeres adultas. Investigación en Enfermería: Imagen y Desarrollo. 2009; 11(1): 97-114.

6. Pilatti A, Castillo D, Martínez VM, Acuña I, Godoy JC, Brussino S. Identificación de patrones de consumo de alcohol en adolescentes mediante análisis de clase latentes. Quaderns Psicología. 2010; 12 (1): 59-73.

7. Villegas-Pantoja MA, Alonso-Castillo MM, Alonso-Castillo BA, Guzmán FR. Eventos Estresantes y la Relación con el Consumo de Alcohol y Tabaco en Adolescentes. Cienc. enferm. 2014; XX(1): 35-46.

8. López-Cisneros MA, Luis MAV, Alonso Castillo MM, Alonso Castillo MT, Aguilar LR. Actitud ante el consumo y no consumo de alcohol en estudiantes de preparatoria-México. Rev. Esc Enferm USP. 2013; 47(4): 815-821.

9. Moreno-Murcia JA, Coll DGC, Cervello-Gimeno E. Motivación y salud en la práctica física deportiva: diferencias según el consumo de alcohol y tabaco. Int J Clin Health Psychol. 2008; 8(2): 483-494.

10. Ruiz-Juan F, Cruz-Sánchez E, García-Montes ME. Motivos para la práctica deportiva y su relación con el consumo de alcohol y tabaco en jóvenes españoles. Salud Publica Méx. 2009; 51(6): 496-504.

11. Vaquero CR, Isorna M, Ruiz C. Review about state of alcohol consumption and physical sports practice. J Sport Health Res. 2012; 4(3): 269-288.

12. Navarro EN, González CD, Marcos PPJ, Borges SF, Hernández PA, Vera LJ, et al. Perfiles motivacionales en la actividad física saludable: un estudio desde la perspectiva de la teoría de la autodetermina- 
ción. Actas del XI Congreso Nacional, XI Andaluz y III Iberoamericano de Psicología de la Actividad Física y del Deporte; 5-8 marzo 2008; Sevilla: Universidad Pablo de Olavide; 2008.

13. Deci LE, Ryan MR. The General Causality Orientations Scale: Self-determination in personality. Journal of Research in Personality. $1985 ; 19(2)$ : 109-134.

14. Ries FC. Actividad Físico-Deportiva en adolescentes de Sevilla y Luxemburgo. Influencia de Factores Personales, Parentales y Situacionales percibidos sobre la intención y la práctica [Tesis Doctoral]. [Sevilla]: Secretario de Publicaciones de la Universidad de Sevilla; 2009. 606 p.

15. Mollinedo FE, Trejo PM, Araujo R, Lugo LG. Índice de masa corporal, frecuencia y motivos de estudiantes universitarios para realizar actividad física. Edu Med Sup. 2013; 27(3): 189-199.

16. Bigelow A, Villarruel A, Ronis D. The relationship of alcohol use and physical activity from an ecologic perspective. J Spec Pediatr Nurs. 2014; 19(1): 28-38.

17. Granero-Gallegos A, Gómez-López M, Albrades V, Rodríguez-Suárez N. Motivos para la práctica en el ámbito de la actividad física no competitiva. Espiral. Cuadernos del Profesorado. 2011; 4(7): 15-22.

18. Barba J, Barba FJ, Domínguez G, Sánchez JA. Adolescentes consumo de alcohol y actividad físico-deportiva. Lecturas: Educación Física y Deporte [Internet]. 2004 [citado 20 nov 2014]; 10(70): 112-120. Disponible en: http://www.efdeportes. com/efd70/alcohol.htm

19. Moreno MJ, Cervello GE, Martínez CA. Validación de la Escala de Medida de los Motivos para la Actividad Física-Revisada en españoles: Diferencias por motivos de participación. Anal. psicol. 2007; 23(1): 167-176.

20. Frederick MC, Ryan MR. Differences in Motivation for sport and exercise and their relations with participation and mental health. J Sport Beh. 1993; 16(3): 124-146.

21. Hodge DR, Gillespie DF. Phrase completions: An alternative to likert scales. Soc Work Res. 2003; 27(1): 45-55.

22. Ruiz RA, Ruiz JF, Zamarripa RJ. Alcohol y tabaco en adolescentes españoles y mexicanos y su relación con la actividad físico-deportiva y la familia. Rev Panam Salud Publica. 2012; 31(3): 211-20.

23. Babor TF, Higgins-Biddle JC, Saunders J, Monteiro M. AUDIT. Cuestionario de identificación de los trastornos debido al consumo de alcohol [Internet]. Washington D.C: OMS; 2001 [citado 20 nov 2014]. 40 p. Disponible en: www.who.int/ substance_abuse/activities/en/AUDITmanualSpanish.pdf

24. De la Fuente J, Kershenobich D. El alcoholismo como problema médico. Rev. Fac. Med. (Méx.). 1992; 35(2): 47-51.

25. Mattara FP, Angelo PM, Faria JB, Alvares DB. Confiabilidade do teste de identificação de transtornos debido ao uso de álcool (AUDIT) em adolescentes. SMAD, Rev. Eletrônica Saúde Mental Álcool Drog [Internet]. 2010 [citado 20 nov 2014]; 6(2): 296-314. Disponible en: http://www.revistas.usp.br/smad/article/ view/38718

26. Secretaría de Salud. Reglamento de la Ley General de Salud en materia de Investigación para la Salud. México: Secretaría de Salud; 1987.

27. Consejo Nacional Contra las Adicciones. Encuesta Nacional de Adicciones 2008 [Internet]. México: Instituto Nacional de Salud Pública; 2008 [citado 20 nov 2014]. 168 p. Disponible en: https://www. insp.mx/images/stories/Produccion/ pdf/100722_cp8.pdf

28. Fernández-Artamendi S, Secades-Villa R, Fernández Hermida J, García-Fernández G, García-Rodríguez O. Gender Differences in Early Alcohol and Tobacco Use as a Risk Factor in Spanish Adolescents. Subst Use Misuse. 2013; 48(6): 429-437. 
29. Ruiz TGM, Medina-Mora IME. La percepción de los adolescentes sobre el consumo de alcohol y su relación con la exposición a la oportunidad y la tentación al consumo de alcohol. Salud Ment. 2014; 37(1): 1-8.

30. Trujillo A, Pérez A, Scoppetta O. Influencia de variables del entorno social sobre la ocurrencia de situaciones problemáticas asociadas al consumo de alcohol en adolescentes. Adicciones. 2011; 23(4): 349-356.

31. Valero A, Ruiz F, García ME, Granero A, Martínez AM. Relación entre la práctica de actividad físico-deportiva y el consumo de alcohol de los ciudadanos de más de 14 años. Adicciones. 2007; 19(3): 239250.

32. Moreno JA, Alonso N, Martínez C, Cervelló E. Motivación, disciplina, coeducación, y estado de flow en educación física: Diferencias según satisfacción, la práctica deportiva y la frecuencia de la práctica. Cuadernos de Psicología del Deporte.
2005; 5(1-2): 225-243.

33. Vaquero CR, Isorna M, Ruiz C. Review about state of alcohol consumption and physical sports practice. J Sport Health Res. 2012; 4(3): 269-288.

34. VanKim NA, Nelson LM, Ehlinger E, Lust K, Story M. Understanding Young adult physical activity, alcohol and tobacco use in community colleges and 4-year post-secondary institutions: A cross-sectional analysis of epidemiological surveillance data. BMC Public Health [Internet]. 2010 [citado 20 nov 2014]; 10(1): 208-216. Disponible en: https://www. ncbi.nlm.nih.gov/pubmed/20420678

35. Lema LF, Salazar IC, Varela MT, Tamayo JA, Rubio A, Botero A. Comportamiento y Salud de los Jóvenes Universitarios: Satisfacción con el estilo de vida. Pensamiento Psicológico. 2009; 5(12): 71-88.

36. Ryan RM, Frederick CM, Lepes D, Rubio $\mathrm{N}$, Sheldon KM. Intrinsic Motivation and exercise adherence. Int J Sport Psychol. 1997; 28(1): 335-354. 